\title{
Juvenile psammomatoid ossifying fibroma of the maxilla
}

\author{
Yongseok Kwon, \\ Donghyeok Shin, \\ Jeenam Kim, \\ Myungchul Lee, \\ Hyungon Choi \\ Department of Plastic Surgery, Konkuk \\ University School of Medicine, Seoul, \\ Korea
}

\begin{abstract}
Juvenile psammomatoid ossifying fibroma (JPOF) is a rare, benign, fibro-osseous variant of ossifying fibroma. It exhibits short-term rapid growth and has a high recurrence rate. Herein we describe a case of JPOF of the maxilla that was treated via complete excision utilizing an intraoral approach with immediate reconstruction using an iliac bone graft, in conjunction with a comprehensive review of the literature. A 20-year-old man presented with a mass on his right cheek that he reported had been growing over the last 10 months. In that cheek he had noticed fullness and experienced pressure, tenderness, and fluffiness, with no other ophthalmic or dental symptoms. After clinical, radiological, and histological examinations, the diagnosis was confirmed as JPOF. Surgical excision was performed, followed by immediate reconstruction with an autologous iliac cortical and cancellous bone graft harvested from the right iliac crest under general anesthesia. Good cicatrization of the intraoral surgical wounds and right iliac crest were evident. He was monitored for 6 months after the surgery and exhibited appropriate midfacial contour. There were no signs of recurrence or complications.
\end{abstract}

Keywords: Bone neoplasm / Jaw neoplasm / Ossifying fibroma

\section{INTRODUCTION}

Ossifying fibroma (OF) is a benign fibro-osseous lesion. It is characterized by the presence of well-demarcated borders and cell-rich fibrosis and contains varying amounts of calcified tissues such as bone or cementum, or both. OF is classified into cemento-OF, juvenile trabecular $\mathrm{OF}$ and juvenile psammomatoid OF (JPOF) [1]. JPOF usually occurs before the age of 15 years, but it can also reportedly occur in adults [2-5]. It has a predilection for the paranasal sinuses and can occur in the maxilla, mandible, orbit, fronto-ethmoid complex, and frontal bone [2-8]. The diagnosis of JPOF is based on characteristic clinical manifestations, histological examination, and radiological characteristics. It is usually asymptomatic but short-term

\section{Correspondence: Hyungon Choi}

Department of Plastic Surgery, Konkuk University School of Medicine,

120-1 Neungdong-ro, Gwangjin-gu, Seoul 05030, Korea

E-mail: 20040059@kuh.ac.kr

Received March 26, 2020 / Revised April 21, 2020 / Accepted May 28, 2020 rapid growth of the mass can cause facial asymmetry, and when the maxilla, mandible, or both are affected it can also cause dental symptoms $[2,7,8]$. Histopathological JPOF exhibits dense cellularity with fibrous stroma and psammoma bodies. Radiological examinations have yielded a large spectrum of findings, including locularity, radiodensity, and cortical bone perforation $[2,7]$. Complete removal is the recommended treatment for JPOF because of its aggressive and locally invasive characteristics, but conservative local excision such as curettage, enucleation, or partial excision can be attempted depending on the patient's individual condition $[2,3,9]$.

\section{CASE REPORT}

A 20-year-old man was referred to our clinic by the department of otorhinolaryngology. Ten months prior he had noticed a mass on his right cheek, which was initially tender. Three months thereafter the mass had started increasing in size, and 
he started experiencing fullness, pressure, and fluffiness on the affected cheek, with no other ophthalmic or dental symptoms. His medical history did not reveal the continued use of medications, and there was no apparent history of genetic disease in his family.

On intraoral examination no protrusion, malocclusion, or tooth mobility were evident. Computed tomography (CT), magnetic resonance imaging (MRI), and various laboratory tests were performed to aid the diagnosis. CT depicted a highdensity mass approximately $3.8 \mathrm{~cm}$ in along its longest axis in the right maxillary sinus near the alveolar process (Fig. 1A and C). MRI depicted a mass of the same sized that exhibited T2low and T1-iso-signal intensity with homogeneous enhancement. The results of all laboratory tests were normal. Under local anesthesia, the patient underwent incisional biopsy via the Caldwell-Luc approach to the sinus, and histopathological reports revealed the lesion to be JPOF.

Due to the well-defined and noninvasive nature of the lesion an intraoral approach was utilized for the excision, and immediate reconstruction with an autologous iliac cortical and can- cellous bone graft under general anesthesia was planned. Intraoperatively a well differentiated, bony, protruding, capsuled, roundish fibroma with a diameter of at least $3 \mathrm{~cm}$ was detected attached to the anterior maxillary wall (Fig. 2A). Complete excision was performed using a drill and an oscillating saw, including the excision of surrounding normal bone (Fig. 3). The bony defect was ultimately found to be approximately ovoid in shape, with a longer diameter of $4 \mathrm{~cm}$ and a shorter diameter of $3 \mathrm{~cm}$. Cortical and cancellous bone were harvested from the right iliac crest for reconstruction. The harvested bone was grafted into the location of the bony defect using titanium plates and screws (Fig. 2B). Five days postoperatively the patient was discharged with mild swelling and right hemifacial numbness (Fig. 1B and D). Two months postoperatively the swelling and hemifacial numbness resolved spontaneously. The diagnosis was confirmed via definitive histopathological examination (Fig. 4). The patient was monitored for 6 months after surgery, and appropriate midfacial contour was maintained. There were no signs of recurrence or complications.
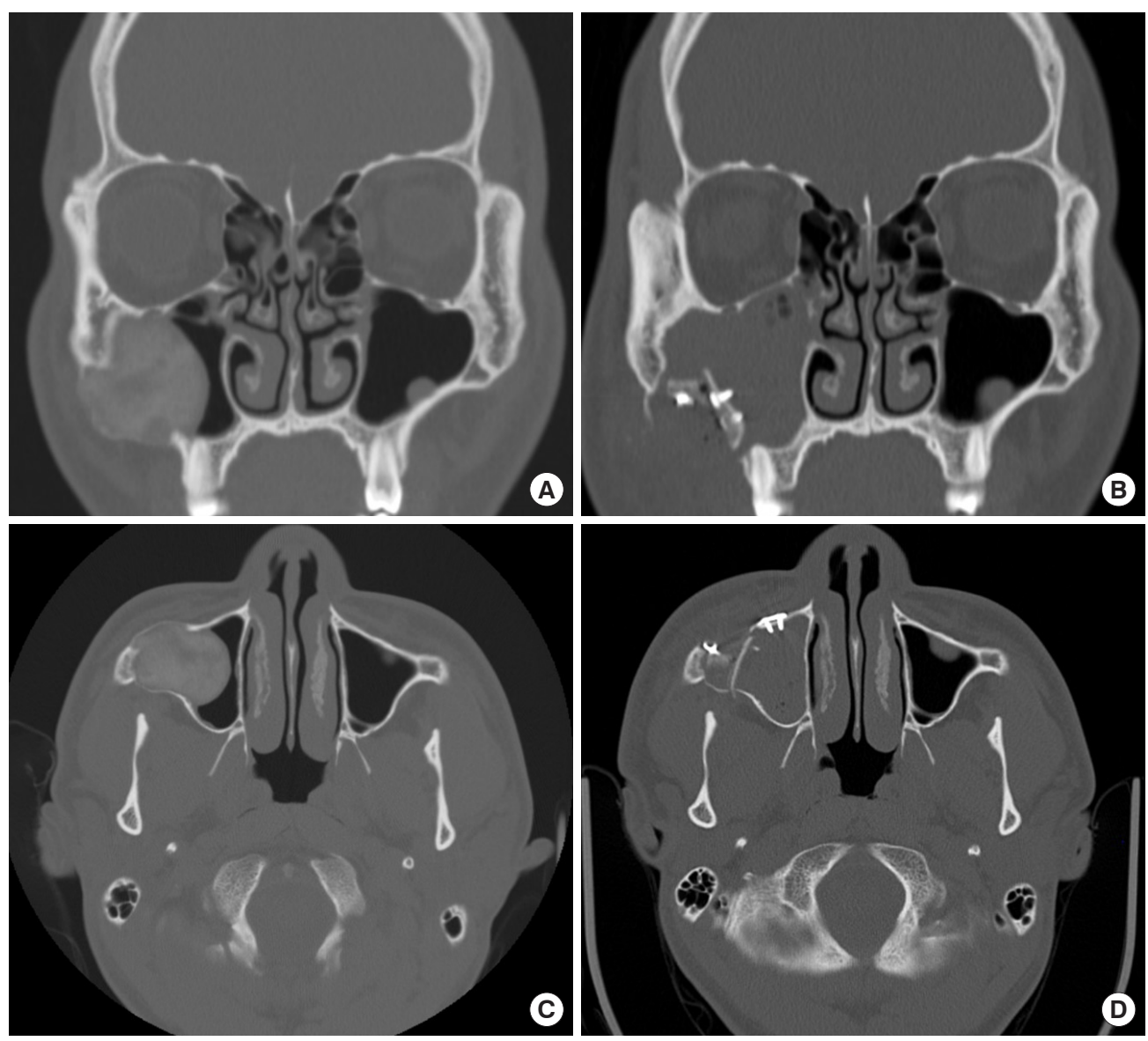

Fig. 1. Facial computed tomography images showing a high-density mass approximately $3.8 \mathrm{~cm}$ in along its longest axis in the right maxillary sinus near the alveolar process. (A) Preoperative coronal view. (B) Coronal view on postoperative day 3. (C) Preoperative axial view. (D) Axial view on postoperative day 3 . 

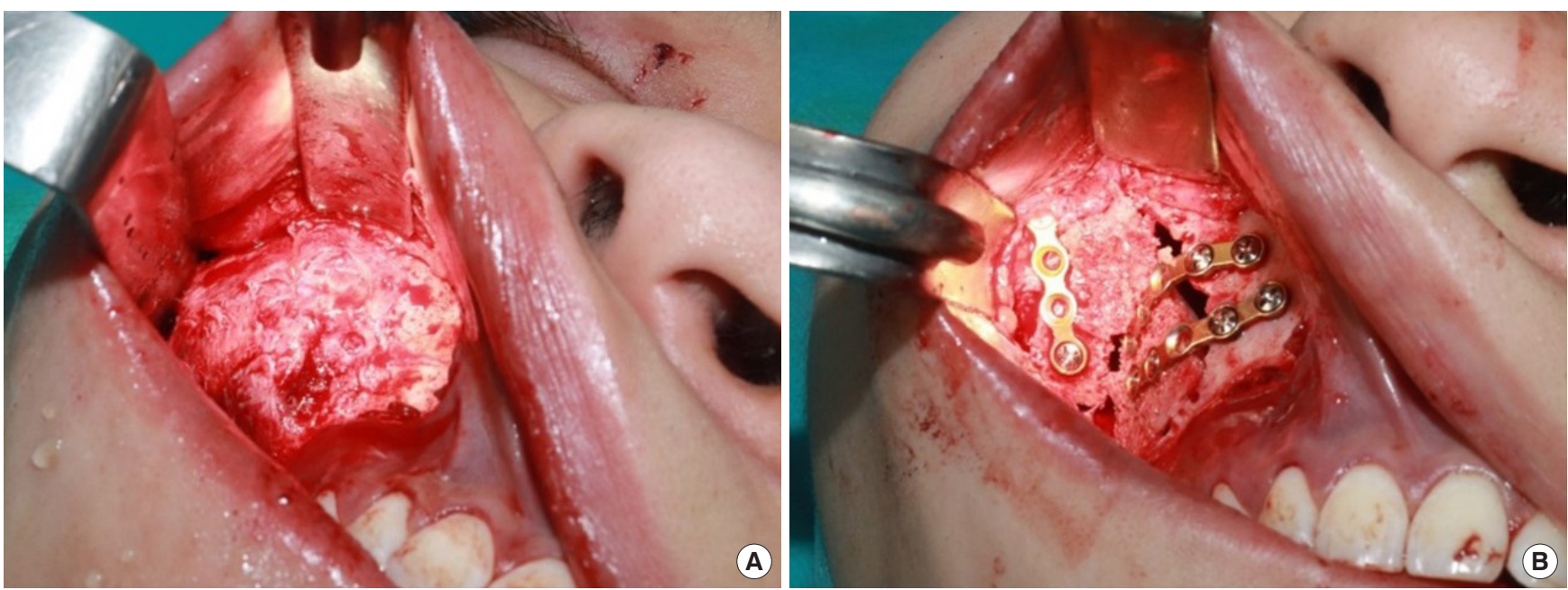

Fig. 2. Intraoperative photographs with intraoral approach. (A) Preoperative photograph. (B) Immediate postoperative photograph.

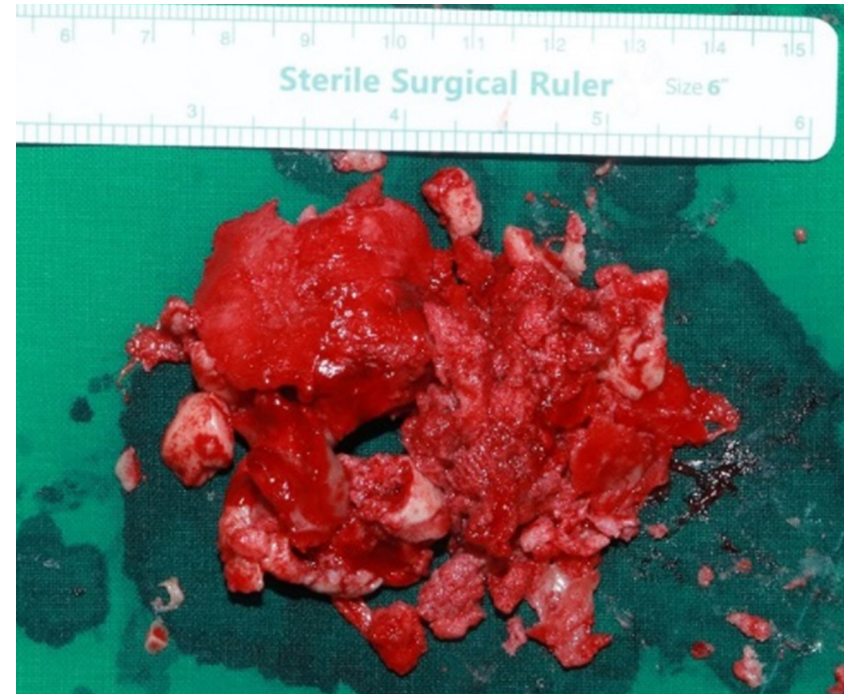

Fig. 3. Gross inspection of excised mass in fragments.

\section{DISCUSSION}

$\mathrm{OF}$ is a benign neoplasm that is classified as central or peripheral depending on its location. Central $\mathrm{OF}$ is further categorized as either cemento-ossifying or juvenile, and juvenile $\mathrm{OF}$ (JOF) is further classified as trabecular or psammomatoid depending on clinical and pathological features $[1,10]$. Cemento$\mathrm{OF}$ is characterized by the presence of a slow-growing mass that expands bone. The mass is usually asymptomatic and rarely recurs. In contrast, JOF exhibits rapid short-term growth [1]. JPOF usually occurs in adolescence, but adults can also be affected [2-5]. In general, age at the time of JPOF onset (3 months to 72 years) is later than that at the time of juvenile trabecular OF (16 to 33 years) [1]. The most common site of JPOF is the paranasal sinuses, followed by the maxilla, mandible, and cal-

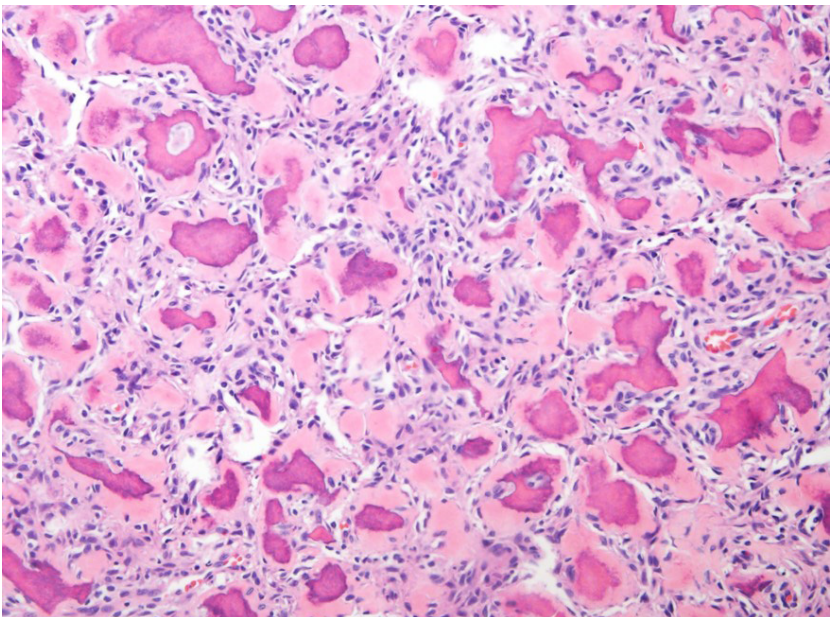

Fig. 4. Photomicrograph depicting numerous small, variably shaped ossicles embedded in fibrous stroma without anaplasia or necrosis (H\&E, $\times 200)$.

varia $[1,10]$. The most common symptoms in patients with JPOF include ocular proptosis, reduced vision, headaches, swelling, stuffiness, nasal obstruction, recurrent sinusitis, and missing teeth, which could be attributed to mass effects $[2,4,5,8]$. In the current patient, there were no symptoms other than local mass effects, such as facial asymmetry.

As JPOF shares many features with other fibro-osseous lesions, its diagnosis should be based on the triad of clinical, radiographic, and histological examinations. The gross macroscopic features of JPOF lesions are a firm to hard consistency and a tan to yellow color, seemingly well-demarcated from the surrounding bone. Radiographic findings are variable, depending on the soft and hard tissue components. CT images depict abnormal bone that differs from the surrounding well-differentiated bony structures. On MRI it resembles a cystic mass with 
an outer hypo-intense shell, suggestive of osteoid matrix calcification. It appears hypo-intense compared to muscle on T1weighted and T2-weighted images. Gadolinium enhancement of the bony wall suggests the absence of reactive hyperostosis $[1,3]$. Histologically JPOF exhibits psammoma bodies, which are concentric or lamellar ossicles. These psammomatoid ossicles contain osteocytes, suggesting an osteogenic origin [11]. In the present patient, the clinical, radiological, and histological characteristics were consistent with JOF with respect to the young age, rapid growth rate, and well-defined radiographical and histological features.

Due to the aggressive characteristics and high recurrence potential of JOF, the standard treatment is complete excision with a safe margin. In cases of large tumors, broad incisions are required to expose the tumor [8]. Therefore, complete resection could prove challenging in terms of reconstruction of the continuity defect, both esthetically and functionally. Depending on the lesion, minimally invasive surgery may be possible via smaller incisions using an intraoral approach or endonasal endoscopy $[2,3,7]$. If the lesion has clear borders with limited range, conservative methods such as curettage, enucleation, or partial resection can achieve acceptable results. Minimally invasive surgeries can effectively conserve adjacent vital structures, particularly in growing patients. One disadvantage of smaller access incisions is the difficulty of complete tumor removal with safe margins, particularly in cases involving more extensive and invasive lesions. The high rate of recurrence of JPOF can lead to a need for repeat surgeries [3].

Although there is no consensus on the optimal method or timing of reconstruction of postoperative defects such as that in the present case, autologous bone grafts are considered the gold standard, with or without the use of a microsurgical technique [8]. Autologous bone grafts provide structural stability, reconstruct the bony defect, and augment bone healing. The properties of these bone grafts include a combination of osteoconductive, osteoinductive, and osteogenic effects. Additionally, there are several donor site options such as the calvaria, iliac crest, fibula, scapula, radius, and ribs. Among these, the iliac crest is the most commonly used donor site for cancellous and corticocancellous grafts [12]. There are also many options with respect to alloplastic materials to use in the reconstruction. Alloplastic materials such as polyethylene, polyamide, metallic mesh (e.g., titanium), or calcium phosphate (e.g., hydroxyapatite) can be used for paranasal sinus reconstruction. Notably however, they are associated with risks of complex reactions with adjacent tissue and of postoperative infection [13]. In order to avoid these disadvantages, in the current case autologous reconstructive surgery was utilized. The lesion was well-differentiated, and the surrounding soft tissues were intact and well vascularized. The maxillary buttress area scheduled for reconstruction was expected to withstand enough force to accommodate mastication. As vascularized bone grafts have the disadvantage of being bulky, a free iliac bone graft was used [14]. Reconstruction can be performed immediately or later, as in a multistage reconstruction. In growing patients, reconstruction should be delayed until the cessation of growth. In some cases, in which the remaining JPOF is stable and can be preserved safely, reconstruction may be not performed at all $[2,8]$.

Postoperative recurrence rates of JPOF range from $30 \%$ to $56 \%$. No malignant transformations have been reported in the literature [3,11]. JPOF shares considerable similarities with other benign fibrous lesions with regard to clinical, radiological, and histopathological features. Accordingly, an adequate understanding of characteristic features of JPOF can facilitate accurate diagnosis, optimal treatment, and excellent prognosis.

\section{NOTES}

\section{Conflict of interest}

No potential conflict of interest relevant to this article was reported.

\section{Ethical approval}

The study was approved by the Institutional Review Board of Konkuk University Medical Center (IRB No. KUMC 2020-03003) and performed in accordance with the principles of the Declaration of Helsinki. Written informed consent was obtained.

\section{Patient consent}

The patient provided written informed consent for the publication and the use of his images.

\section{ORCID}

Yongseok Kwon https://orcid.org/0000-0003-4584-797X

Donghyeok Shin https://orcid.org/0000-0002-8450-4411

Jeenam Kim https://orcid.org/0000-0002-4080-6135

Myungchul Lee https://orcid.org/0000-0002-9721-0092

Hyungon Choi https://orcid.org/0000-0002-3816-1286

\section{REFERENCES}

1. Hameed M, Horvai AE, Jordan RCK. Soft tissue special issue: gnathic fibro-osseous lesions and osteosarcoma. Head Neck Pathol 2020;14:70-82.

2. Diniz JA, Siqueira ADS, Araujo GM, Faro TF, Torres LHS, 
Oliveira E Silva ED, et al. Intraoral approach for surgical treatment of psammomatoid juvenile ossifying fibroma. J Craniofac Surg 2020;31:e306-9.

3. Kim DY, Lee OH, Choi GC, Cho JH. A case of juvenile psammomatoid ossifying fibroma on skull base. J Craniofac Surg 2018;29:e497-9.

4. Martinez-Manas RM, Rey MJ, Gaston F. Aggressive psammomatoid ossifying fibroma. Ann Otol Rhinol Laryngol 2002; 111(5 Pt 1):466-8.

5. Yang HY, Zheng LW, Luo J, Yin WH, Yang HJ, Zwahlen RA. Psammomatoid juvenile cemento-ossifying fibroma of the maxilla. J Craniofac Surg 2009;20:1190-2.

6. Wehrli LA, Zweifel N, Weil R, Altermatt S. Juvenile psammomatoid ossifying fibroma of the forehead, radical resection, and defect coverage with a hydroxyl-apatite composite: a case report. Eur J Pediatr Surg 2012;22:479-84.

7. Gantala R, Vemula AY, Kubbi JR, Sekhar MM, Jhawar D. Psammomatoid juvenile ossifying fibroma involving upper jaw: a rare case report. J Clin Diagn Res 2015;9:ZD17-9.

8. Wong WW, Martin MC. Reconstruction of extended orbitomaxillectomy and hemimandibulectomy defects with fibula flaps and patient-specific implants. J Craniofac Surg 2016;27: 380-4.

9. Slootweg PJ, Muller H. Juvenile ossifying fibroma: report of four cases. J Craniomaxillofac Surg 1990;18:125-9.

10. Eversole R, Su L, ElMofty S. Benign fibro-osseous lesions of the craniofacial complex: a review. Head Neck Pathol 2008;2:177202.

11. Wenig BM, Vinh TN, Smirniotopoulos JG, Fowler CB, Houston GD, Heffner DK. Aggressive psammomatoid ossifying fibromas of the sinonasal region: a clinicopathologic study of a distinct group of fibro-osseous lesions. Cancer 1995;76:115565.

12. Myeroff C, Archdeacon M. Autogenous bone graft: donor sites and techniques. J Bone Joint Surg Am 2011;93:2227-36.

13. Eppley BL. Alloplastic biomaterials for facial reconstruction. In: Booth PW, Eppley BL, Schmelzeisen R, editors. Maxillofacial trauma and esthetic facial reconstruction. 2 nd ed. St. Louis: Elsevier/Saunders; 2012. p. 132-43.

14. Davison SP, Mesbahi AN, Clemens MW, Picken CA. Vascularized calvarial bone flaps and midface reconstruction. Plast Reconstr Surg 2008;122:10e-18e. 